\title{
EVIDENCE FOR RELATIVISTIC MOTION BASED ON ARCSECOND STRUCTURE ${ }^{+}$
}

\author{
R. A. Perley \\ National Radio Astronomy Observatory, Socorro, U.S.A.
}

\begin{abstract}
Observations with arcsecond-resolution interferometers can be used to detect motion or changes of the large scale structure associated with core dominated objects. If these arcsecond structures are expanding at relativistic velocities, detectable changes should be measureable within a few years. Indirect estimates of jet velocities can be made by a number of arguments, but none are model independent or free of dependence upon poorly established parameters. Application of some of these methods are given on sources with established VLB structure.
\end{abstract}

1. Direct Measurement of Proper Motion. Nearly all the known superluminal sources contain arcsecond structures located on the same side as the relativistic jet, an observation which suggests that the arcsecond structure is being continually fed by the milliarcsecond jet. Generally, the arcsecond structures are not smooth, but contain bright knots of considerable brightness gradient. Are the knots moving or changing in a detectable way? Since the cores of these sources provide a reliable phase and amplitude reference, this question may be answered in a reasonable time scale through observations with instruments such as the VLA. Such a detection will not necessarily give the jet velocity - the feature may be a slower moving shock or density enhancement (e.g. Blandford and Konigl, 1979). Noting that it is in regions of strong brightness contrast that the most sensitive measurements of change of

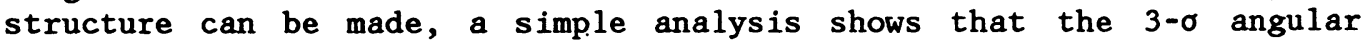
change, $\Delta d$, that can be measured is:

$$
\Delta d=2 \sigma / s \text {, }
$$

where $\sigma$ is the rms noise on the map, and $s$ is the maximum brightness slope (seen by the instrument) of the knots whose motion or change we wish to measure.

Ron Ekers and I have initiated an experiment on the VLA to perform this measurement on the sources $3 \mathrm{C} 273$ and $3 \mathrm{C} 279$, both of which contain arcsecond jets with considerable brightness enhancements. At this time, only first epoch observations have been made, so no results are yet available. From these first-epoch maps, we estimate that for $3 \mathrm{C} 279$ at $2 \mathrm{~cm}$, a motion as small as 8 milliarcseconds can be detected. With careful

+ Discussion on page 438 153

R. Fanti et al. (eds.), VLBI and Compact Radio Sources, 153-156.

(C) 1984 by the IAU. 
data editing and map analysis, this limit should drop considerably. The theoretical limit (using thermal noise in 12 hours) predicts than ar: angular discrimination of only 0.4 marc should be possible. For 3C273, the current maps indicate an angular change discrimination of better than 4 marc, or 2.5 marc using the polarized flux (where dynamic range problems are less severe). The theoretical limit for this source is as low as 0.1 marc. Note however, that better than $60 \mathrm{~dB}$ dynamic range will be required to reach this limit. Current best dynamic ranges are 40 to $45 \mathrm{~dB}$ - we do not fully understand why we cannot reach higher dynamic ranges.

This method should be applicable to any source with a strong, unresolved core and arcsecond structure with good brightness contrast.

2. Periodicities in structure. The curvature seen in VLB maps of the jets in core-dominated sources (e.g. 3C 345, 3C418, 3C273, VLB maps of which have been shown in this conference) lends credence to the idea that a precessing collimator is operating in the core. If, in fact, this is occuring, we might expect the arcsecond structures to reflect the periodicity set by the collimator. Fig 1 . shows a $6 \mathrm{~cm}$ VLA map with $0.6^{\prime \prime}$ resolution of the arcsecond component of $3 \mathrm{C} 273$.

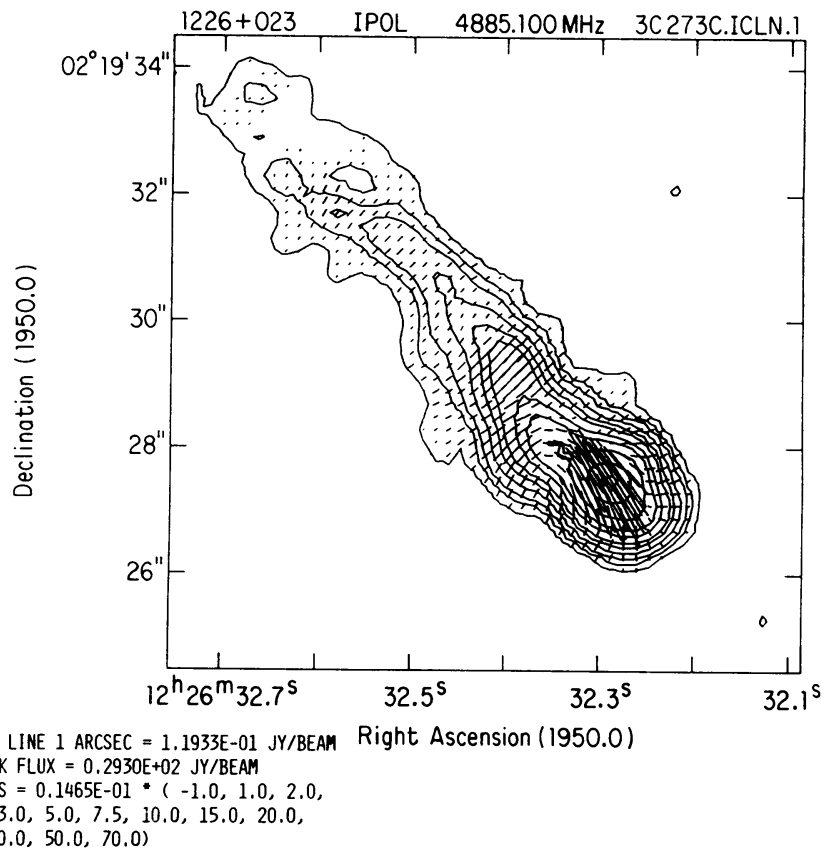

Fig. 1. The arcsecond jet of $3 \mathrm{C} 273$ at $6 \mathrm{~cm}$ with 0.66 resolution. The intensity and direction of the polarized flux are shown by the dashes.

Note the sinusoidal shape of the ridge of maximum brightness. If this is due to the collimator (rather than a hydrodynamic instability as suggested 
by Conway et al., 1981), a direct measure of the jet velocity, $v_{j}$, is possible:

$$
v_{j}=L_{o}(1-\beta \cos \theta) \csc \theta / T_{c} .
$$

Here, $L_{o}$ is the apparent spatial period, $T_{c}$ the precession period, $\beta=$ $v_{j} / c$, and $\theta$ is the angle of jet motion from the line of sight. Noting in Fig 1 that the jet ridge line is remarkably sinusoidal for a jet which is probably greatly foreshortened, a geometrical argument can be used from which the following equation arises:

$$
v_{j}>40 R(1-\beta \cos \theta) \operatorname{cotan} \theta / T_{c} .
$$

Here, $R$ is the radius of the channel into which the jet is wound, and other symbols are as before. Inserting measured values into this latter equation yields :

$$
\beta>\left(\mathrm{T}_{\mathrm{p} 5} \tan \theta+\cos \theta\right)^{-1} \text {. }
$$

Here, $T_{p 5}$ is the precession period in units of $10^{5}$ years. If $\theta<10$ degrees, as is probable, then $\beta$ is relativistic if the precession period is less than $10^{5}$ years.

3. Brightness Ratios This argument is applicable if jets are oppositely directed and equal in their respective frames (i.e. each evolves and radiates equally). Then, the measured flux ratio between knots identified as having been ejected at the same time is:

$$
\mathrm{F}^{+} / \mathrm{F}^{-}=[(1+\beta \cos \theta) /(1-\beta \cos \theta)]^{3-\alpha} \text {. }
$$

For intensity ratios of extended objects, the index should be $2+\alpha$ (e.g. Blandford and Konigl, 1979). The problem with this approach, besides the assumption of symmetry, is that the ratio is such a strong function of the index that mildly relativistic motion will lead to flux ratios beyond the dynamic range of current instruments. For example, in 3C273, the flux ratio is $>500: 1$ - leading to $\beta \cos \theta>0.67$ - not an impressive limit.

4. Jet/Counterjet Symmetry. This argument again requires the jets to be intrinsically identical and oppositely directed. Then, for ballistic motion (or non-balistic motion through identical media with no disruptive instabilities), any feature on the approaching jet should be mirrored on the receding jet, but due to time-delay effects, the location of the feature is closer to the nucleus. The ratio between approaching and receding separations is:

$$
\theta^{+} / \theta^{-}=(1+\beta \cos \theta) /(1-\beta \cos \theta) \text {. }
$$

For the velocity limit given above for $3 \mathrm{C} 273$, this equation predicts the counter-blob (if it exists at all) should be within $4.5^{\prime \prime}$ of the nucleus.

The spectacular jet in NGC6251 provides an interesting test of these last two relationships. The jet/counter-jet brightness ratio leads to the limit $\beta \cos \theta>0.6$. However, new VIA maps show that the ratio of angular separations for prominent bends in the jets is less than 1.1 , leading to $B \cos \theta<0.05$. Clearly, both velocity limits cannot apply, so one of our initial assumptions is wrong, most likely that the jets are radiating equally in their own frames. This provides important evidence that other factors are of great importance in the determination of jet brightness. 
5. Global Lobe Properties. In this approach, one attempts to estimate the properties of the jet from known properties of the lobes which the jets feed with energy, momentum and mass. The transport equations for a relativistic jet of cross-section $A$, mass density $\rho$, internal energy density $\mu$, velocity $\beta=v / c$ and Lorentz factor $\gamma$ are:

Kinetic Energy: $\gamma^{2} \mathrm{Av}^{3} p\left[\gamma /(\gamma+1)+\alpha / \beta^{2}\right]$, where $\alpha=\mu /\left(\rho c^{2}\right)$.

Momentum $\quad A \rho v^{2} \gamma^{2}$.

Mass Flux Apv $\gamma$.

We can estimate the K.E. flux from the (roughly) known total luminosity of the lobe which the jet is supposed to feed, the momentum flux can be estimated from the familiar argument of the required thrust for the jet to enter the hot spot where the jet is supposed to terminate (using the usual equipartition method), while, at least in principle, the mass flux can be derived from the total mass (from depolarization), and source age (from spectral steepening, or some other way.)

Unfortunately, none of the above parameters can be estimated with any degree of accuracy. The K.E. flux of the jet goes to other sinks besides the radiated luminosity (see Saunders et al., 1981 for a discussion), the thrust argument surely provides a lower limit to the momentum, while interpretation of depolarization data (if it exists at all) is fraught with problems (see Perley et al., 1983, for a discussion). Even if any one of these global quantities could be estimated accurately, the problem of the jet density remains. This vital quantity is completely unknown, and dependable measures of it remain a long way off. Independence from this parameter can be gained by taking ratios amongst the equations however, this requires ratios of the poorly determined global parameters as well, with the usual accompanying deterioration of accuracy. Thus, it appears this approach to measuring jet velocities is not a fruitful one.

Recently, a jet in Cygnus $A$ has been discovered (Perley and Cowan, in preparation). Application of these ideas to Cygnus A ( a source whose global prameters are known better than most) leads to only one firm conclusion - that the jet in Cygnus A cannot have an internal energy density exceeding the dynamic pressure $\rho v^{2}$ and be relativistic - for this case cannot provide enough thrust for the jet to terminate in the hot spots found at the ends of the source. To make use of the global approach, much better measures of the energy, momentum and mass of the lobes will be needed.

\section{REFERENCES}

Blandford, R.D., and Konig1, A. Ap.J. 232, 34 (1979)

Conway, R.G., Davis, R.J., Foley, A.R., and Ray, T.P. Nature 294, 540 (1981)

Perley, R.A., Bridle, A.H., and Willis, A.G. Submitted to Ap. J.

Saunders, R., Baldwin, J.E., Pooley, G.G., and Warner, P.J. MNRAS, 197, 253 (1981). 